SHORT REPORT

\title{
Mannan binding lectin in febrile adults: no correlation with microbial infection and complement activation
}

\author{
A N Tacx, A B J Groeneveld, M H Hart, L A Aarden, C E Hack
}

J Clin Pathol 2003;56:956-959

\begin{abstract}
Aims: To study the role of the mannan binding lectin (MBL) pathway of complement activation in the host defence to microbial infection in vivo, and the role of $M B L$ in infectious mortality in non-selected patients.

Methods: A prospective observational study on 177 hospitalised medical patients with new onset fever. The presence, origin, and microbial cause of infection, the circulating MBL and complement activation product $3 a(C 3 a)$, and the 28 day hospital course were determined.

Results: The patients had median MBL values similar to healthy blood donors: $18 \%$ of the patients and $14 \%$ of the blood donors had MBL deficiency, with values below $0.1 \mu \mathrm{g} /$ $\mathrm{ml}$. Median C3a was higher in patients with microbiologically confirmed infection than in those without, whereas there was no difference in $\mathrm{MBL}$ values or frequency of deficiency among patient groups with or without positive local cultures or bacteraemia. The mortality rate was $8 \%$ and the outcome groups did not differ in MBL. In febrile adults hospitalised in internal medicine wards, microbial infection induces complement activation, independently of MBL.

Conclusions: The results argue against a predominant role for the MBL pathway of complement activation and a deficiency of $M B L$ predisposing to serious and invasive microbial infection in non-selected adults.
\end{abstract}

$\mathrm{T}$ here are numerous causes of immunodeficiency that predispose to bacterial infections. A recently discovered factor is deficiency of the lectin pathway of complement activation. ${ }^{12}$ Indeed, genetic and phenotypic deficiency in producing mannan binding lectin (MBL) may predispose to unusual, recurrent or serious infections in selected groups of patients-for instance, in children and patients with neutropenia or haematological cancer-presumably by attenuating complement activation and opsonisation, as suggested by in vitro experiments. ${ }^{1-8}$ To study this relation in non-selected patients, we determined circulating MBL and complement activation product $\mathrm{C} 3 \mathrm{a}$ in 177 consecutive patients with fever on general internal medicine wards, and studied their interrelation and association with microbial infection and mortality.

"Genetic and phenotypic deficiency in producing mannan binding lectin may predispose to unusual, recurrent or serious infections in selected groups of patients"

\section{PATIENTS AND METHODS}

During a one year period, 300 consecutive patients with new onset of fever (body temperature $>38.0^{\circ} \mathrm{C}$ axillary or $38.3^{\circ} \mathrm{C}$ rectal) admitted to the department of internal medicine of a university hospital, were included in our study. Some of the data collected in this cohort have been published previously. ${ }^{9}$ We now report on the 177 patients in whom it was possible to determine MBL at inclusion into our study. The study was approved by the local committee on ethics. All patients or their closest relatives gave informed consent before inclusion. Exclusion criteria were pregnancy, use of cytokines (such as interferon $\gamma$ or interleukin 2), treatment for malignant haematological disease, shock, and a life expectancy of less than 24 hours. Patients were taken care of by attending physicians not involved in our study. They ordered imaging techniques and other tests to establish a clinical focus of infection.

At inclusion, demographics and variables that could influence culture results and the concentrations of inflammatory mediators were recorded, including age, sex, underlying disease (such as infection with human immunodeficiency virus), malignancies, or diabetes mellitus, before treatment with immunocompromising (cytostatic or steroidal) drugs or antibiotics. The time from fever onset to admission was estimated, to separate community acquired from nosocomial fever, which was defined as fever emerging 72 hours or longer after hospital admission. The time from onset of fever to inclusion was also estimated. We measured body temperature and white blood cell counts at inclusion. The development of shock was evaluated during a maximum follow up of seven days, when the patients were still in the hospital. If patients had been discharged within this period, they were classified as not having shock. Shock was defined by hypotension; that is, a systolic blood pressure $<90 \mathrm{~mm} \mathrm{Hg}$ or a reduction of $>40 \mathrm{~mm} \mathrm{Hg}$ from baseline values, in the absence of other causes of hypotension, despite adequate fluid resuscitation, or by the need of administration of vasoactive drugs to increase blood pressure. All cause mortality within 28 days after study inclusion was also recorded. A patient discharged from hospital within this period was regarded as a survivor.

\section{Microbial cultures}

At least two blood samples were obtained by venipuncture at inclusion for microbiological studies, as described previously. ${ }^{9}$ Supplementary blood cultures were taken when clinically indicated. Local specimens for culture were collected by the attending physician, depending on the clinical situation; that is, when a focus of infection was suspected. Results from microbiological studies performed during the first seven days after inclusion were recorded. Patients were classified into groups depending on culture results: patients in group 1 did not have positive cultures; patients in group 2 had positive

Abbreviations: $\mathrm{C} 3 \mathrm{a}$, complement activation product $3 a ; \mathrm{MBL}$, mannan binding lectin 
local cultures or positive specific stains for fungal infections; and patients in group 3 had bacteraemia, with or without local positive cultures. Blood cultures were processed using "delayed vial entry" bottles for aerobic and anaerobic cultures and the Bactec 9120/9240 automatic analysers (Becton Dickinson, Erembodegem, Belgium). Bottles were incubated for a maximum of seven days. If the analysers showed growth, Gram stains were prepared and the sensitivity of the organism for antibiotics was assessed. Blood cultures containing Staphylococcus epidermidis were considered contaminated if only one bottle revealed growth and there were no indwelling vascular catheters. Local cultures were processed according to standardised procedures and, when indicated, specific stains were performed, to document fungal infections. Positive local microbiological results were only taken into account when thought to reflect infection as opposed to colonisation, if the treating physician decided to prescribe or continue antimicrobial drugs based on these results.

Blood samples for the determination of plasma concentrations of inflammatory mediators were obtained at inclusion. The samples were collected in tubes containing soybean trypsin inhibitor $(100 \mu \mathrm{g} / \mathrm{ml}$, final concentration), EDTA ( $10 \mathrm{mmol} / \mathrm{litre})$, and benzamidin ( $10 \mathrm{mmol} /$ litre), to prevent in vitro activation. All tubes were centrifuged for 10 minutes at $1300 \times g$ and aliquots of the plasma were stored immediately at $-70^{\circ} \mathrm{C}$ until assays were performed.

\section{Assays}

Complement activation in the patients was measured by assessing circulating concentrations of $\mathrm{C} 3 \mathrm{a}$ with a radioimmunoassay, as described previously. ${ }^{9}$ Results were expressed as nmol/litre. Normal values in healthy volunteers are less than or equal to $5 \mathrm{nmol} /$ litre. ${ }^{9}$

MBL was determined using an enzyme linked immunosorbent assay in which samples were incubated in mannan coated plates. After washing, binding of MBL was visualised by subsequent incubation with biotinylated monoclonal antibody against MBL (CLB anti-MBL-1). Values below about $0.1 \mu \mathrm{g} / \mathrm{ml}$ point to heterozygous deficiency and undetectable amounts to homozygous deficiency. ${ }^{124710}$ Consecutive healthy blood donors $(\mathrm{n}=99)$, evaluated by the central laboratory of the Dutch Red Cross Blood Transfusion Service (Amsterdam), were used to determine MBL values in the normal population.

\section{Statistical analysis}

Data were summarised as median/range, and $\chi^{2}$ and KruskalWallis analysis of variance tests were used to compare groups. Backward logistic regression was used to find the smallest set of variables predictive for bacteraemia, on the basis of the likelihood ratio. The Hosmer-Lemeshow test was used to evaluate the goodness of fit, with a $p$ value approaching $\mathrm{l}$ indicating a perfect fit of predicted on observed frequencies. $\mathrm{p}<0.05$ was considered significant.

Table 1 Patient characteristics according to microbial infection

\begin{tabular}{|c|c|c|c|c|}
\hline & $\begin{array}{l}\text { Group 1 } \\
(n=115)\end{array}$ & $\begin{array}{l}\text { Group } 2 \\
(n=43)\end{array}$ & \multicolumn{2}{|l|}{$\begin{array}{l}\text { Group } 3 \\
(n=19)\end{array}$} \\
\hline Age (years) & $63(17-88)$ & $66(24-97)$ & \multicolumn{2}{|l|}{$71(21-83)$} \\
\hline Sex (male/female) & $65 / 50$ & $20 / 23$ & \multicolumn{2}{|l|}{$6 / 13$} \\
\hline Non whites & $8(7 \%)$ & $2(5 \%)$ & \multicolumn{2}{|l|}{$1(5 \%)$} \\
\hline Underlying disease & & & \\
\hline Cardiovascular & $27(23 \%)$ & $10(23 \%)$ & & \\
\hline Respiratory & $14(12 \%)$ & $4(9 \%)$ & $1(5 \%)$ & \\
\hline Urogenital & $16(14 \%)$ & $8(19 \%)$ & \multicolumn{2}{|l|}{$4(21 \%)$} \\
\hline Gastrointestinal & $4(3 \%)$ & $4(9 \%)$ & \multicolumn{2}{|l|}{$1(5 \%)$} \\
\hline Neurological & $12(10 \%)$ & $6(14 \%)$ & \multicolumn{2}{|l|}{$1(5 \%)$} \\
\hline Diabetes mellitus & $15(12 \%)$ & $8(19 \%)$ & \multicolumn{2}{|l|}{$6(32 \%)$} \\
\hline HIV/AIDS & $7(5 \%)$ & $1(2 \%)$ & & \\
\hline Cancer & $27(23 \%)$ & $7(16 \%)$ & \multicolumn{2}{|l|}{$4(21 \%)$} \\
\hline Autoimmune disease & $9(8 \%)$ & $5(12 \%)$ & \multicolumn{2}{|l|}{$3(16 \%)$} \\
\hline Recent treatment & & & \\
\hline Recent surgery & $15(13 \%)$ & $4(9 \%)$ & & \\
\hline Immunosuppressive treatment & $23(20 \%)$ & $8(19 \%)$ & \multicolumn{2}{|l|}{$2(11 \%)$} \\
\hline Previous antibiotic treatment & $21(18 \%)$ & $10(23 \%)$ & \multicolumn{2}{|l|}{$3(16 \%)$} \\
\hline Source of infection & $71(62 \%)^{*}$ & $43(100 \%)^{*}$ & \multicolumn{2}{|l|}{$18(95 \%)^{*}$} \\
\hline Cardiovascular & 1 & 1 & \multirow{2}{*}{\multicolumn{2}{|c|}{3}} \\
\hline Lung & 41 & 21 & & \\
\hline Urinary tract & 10 & 14 & \multicolumn{2}{|l|}{5} \\
\hline Soft tissue & 4 & 7 & \multicolumn{2}{|l|}{8} \\
\hline CNS & 2 & & \\
\hline Gastrointestinal tract & 12 & & \multicolumn{2}{|l|}{2} \\
\hline Intravascular catheter & 1 & & & \\
\hline Associated microorganism & & Local $^{*}$ & \multirow{5}{*}{\multicolumn{2}{|c|}{$\begin{array}{l}\text { Blood* } \\
11(58 \%) \\
6(32 \%) \\
2(11 \%)\end{array}$}} \\
\hline Gram positive & & $5(12 \%)$ & & \\
\hline Gram negative & & $24(56 \%)$ & & \\
\hline Polybacterial & & $7(6 \%)$ & & \\
\hline Positive fungal stain & & $7(6 \%)$ & & \\
\hline Community acquired fever & & $84(73 \%)$ & \multirow{6}{*}{$\begin{array}{l}33(77 \%) \\
14(0.1-1226) \\
38.8(38.2-41.3) \dagger \\
12.2(0.3-25.8) \\
2(5 \%) \\
1(2 \%) \ddagger\end{array}$} & $14(74 \%)$ \\
\hline Time to inclusion (hours) & & $12(0.5-575)$ & & $11(1-130)$ \\
\hline Temperature $\left({ }^{\circ} \mathrm{C}\right)$ & & $38.9(38.0-40.7) \dagger$ & & $39.3(38.4-40.1) \dagger$ \\
\hline WBC ( $\left.\times 10^{9} / \mathrm{I}\right)$ & & $11.1(0.3-42.3)$ & & $11.8(3.2-27.2)$ \\
\hline Leucopenia $\left(<1.0 \times 10^{9} / \mathrm{l}\right)$ & & $5(4 \%)$ & & \\
\hline Shock & & & & $2(11 \%) \ddagger$ \\
\hline Mortality & & $7(6 \%)$ & \multicolumn{2}{|l|}{$7(16 \%)$} \\
\hline $\begin{array}{l}\text { Values are median (range) or nu } \\
\text { Group 1, culture negative; group } \\
\text { positive local cultures }(n=12) \text {. } \\
\text { CNS, central nervous system; HII } \\
{ }^{*} p<0.001 \text { for frequency and dis } \\
\text { (Kruksal-Wallis test); } \neq p<0.005\end{array}$ & $\begin{array}{l}\text { umber (percent } \\
\text { p 2, local cultu } \\
\text { IV, human imm } \\
\text { stribution of sou } \\
\text { ( } \chi^{2} \text { test). }\end{array}$ & $\begin{array}{l}\text { tage), where appropr } \\
\text { rre positive; group } 3, \\
\text { unodeficiency virus; } \\
\text { urces and microorgan }\end{array}$ & $\begin{array}{l}\text { iate. } \\
\text { bacteraemia ( } n=7 \text { ) or } \\
\text { WBC, white cell count. } \\
\text { isms between groups }\end{array}$ & $\begin{array}{l}\text { bacteraemia and } \\
\left(\chi^{2} \text { test }\right) ; \mathrm{tp}<0.05\end{array}$ \\
\hline
\end{tabular}




\section{RESULTS}

\section{Patients}

Of the 177 patients, 14 died $(8 \%)$. Mortality did not differ significantly among the three groups. Table 1 provides the demographic and clinical features. The time to inclusion was variable, for both community acquired and hospital acquired fever.

\section{Infection}

One hundred and fifty one (85\%) patients had a clinical source of infection, $62(35 \%)$ a positive microbiological result, and $17(11 \%)$ bacteraemia (table 1$)$. Otherwise, in patients without positive cultures, there was a clinical infection source presumed in only $62 \%$ of patients. All patients with positive cultures, except for one patient with bacteraemia, had a presumed clinical focus.

\section{$M B L$ and $C 3 a$}

In healthy blood donors, the MBL was below $0.1 \mu \mathrm{g} / \mathrm{ml}$ in $14 \%$ of patients, with a median value of 1.4 and a range of $0-$ 33. Thirty two $(18 \%)$ of the patients had MBL values below $0.1 \mu \mathrm{g} / \mathrm{ml}$, which did not differ from the frequency in healthy blood donors (table 2). In $142(80 \%)$ patients, C3a was above the upper limit of normal ( $5 \mathrm{nmol} /$ litre). There was no difference in C3a concentrations between patients with MBL values below and above $0.1 \mu \mathrm{g} / \mathrm{ml}$ or below or above $1.5 \mu \mathrm{g} /$ $\mathrm{ml}$. The concentration $1.5 \mu \mathrm{g} / \mathrm{ml}$ was arbitrarily chosen because MBL may behave as an acute phase protein. Hence, there was no correlation between MBL and C3a concentrations (fig 1). However, table 2 shows that C3a concentrations were significantly higher in culture positive versus culture negative patients, whereas MBL concentrations were not. Female sex, presence of a clinical infectious focus, and a raised C3a concentration significantly $(\mathrm{p}<0.05)$ predicted bacteraemia, whereas age, diabetes, temperature, WBC count, and MBL did not contribute independently (Hosmer Lemeshow $\chi^{2}=10.8$; degrees of freedom, $8 ; p=0.22$ ). There was no difference in the frequency of $\mathrm{MBL}<0.1 \mu \mathrm{g} / \mathrm{ml}$ among the three groups.

\section{Outcome}

Groups differed in occurrence of shock but not in ultimate outcome, so that bacteraemia was associated with shock development but not necessarily with a poor outcome. Conversely, the three shock patients had a positive microbiological result and two had bacteraemia, so that the patients suffered from septic shock. The MBL concentrations in the patients with septic shock ranged from 0.1 to $6.1 \mu \mathrm{g} /$ $\mathrm{ml}$. There was no difference in MBL values between dying and surviving patients, because an $\mathrm{MBL}<0.1 \mu \mathrm{g} / \mathrm{ml}$ occurred in $17 \%$ (28 of 163 ) of the survivors and $28 \%$ (four of 14 ) of the non-survivors (not significant).

Table 2 Circulating mannan binding lectin (MBL) and $\mathrm{C} 3 \mathrm{a}$ in febrile medical patients, according to microbial infection

\begin{tabular}{llll}
\hline & $\begin{array}{l}\text { Group 1 } \\
(\mathbf{n}=115)\end{array}$ & $\begin{array}{l}\text { Group 2 } \\
(\mathbf{n}=43)\end{array}$ & $\begin{array}{l}\text { Group 3 } \\
(\mathbf{n}=19)\end{array}$ \\
\hline $\mathrm{MBL}(\mu \mathrm{g} / \mathrm{ml})$ & $1.1(0-12.3)$ & $1.7(0-10.0)$ & $2.5(0-6.4)$ \\
$\mathrm{MBL}<0.1 \mu \mathrm{g} / \mathrm{ml}$ & $21(18 \%)$ & $9(21 \%)$ & $2(11 \%)$ \\
$\mathrm{MBL}>1.5 \mu \mathrm{g} / \mathrm{ml}$ & $53(46 \%)$ & $23(53 \%)$ & $12(63 \%)$ \\
$\mathrm{C} 3 \mathrm{a}(\mathrm{nmol} / \mathrm{l})$ & $7.4(2.3-35.0)^{*}$ & $8.3(2.4-21.0)^{*}$ & $8.1(6.3-52.0)^{*}$ \\
$\mathrm{C} 3 \mathrm{a}>5 \mathrm{nmol} / \mathrm{l}$ & $86(75 \%) \dagger$ & $37(86 \%) \dagger$ & $19(100 \%) \dagger$ \\
\hline
\end{tabular}

Values are median (range) or number (percentage), where appropriate. Group 1, culture negative; group 2, local culture positive; group 3, bacteraemia $(n=7)$ or bacteraemia and positive local cultures $(n=12)$. ${ }^{*} \mathrm{p}<0.05$ (Kruskal-Wallis test); $\mathrm{tp}<0.05$ ( $\chi^{2}$ test).

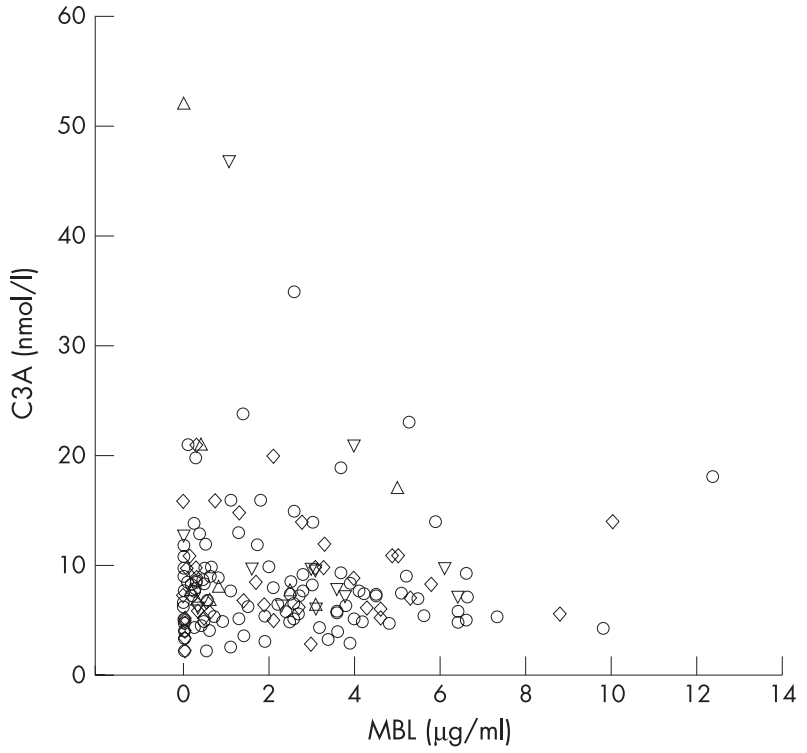

Figure 1 Scatterplot of circulating mannan binding lectin (MBL) versus complement activation product $3 a(C 3 a)$ in patients without positive cultures (circles), with local positive cultures (diamonds), with bacteraemia only (triangles), and with positive local and blood cultures (inverted triangles).

\section{DISCUSSION}

Our results suggest that in febrile medical patients, microbial infection and increasing invasiveness thereof is associated with increasing complement activation in blood, largely independent of MBL.

This is the first study that we are aware of concerning MBL and complement activation in vivo. Indeed, MBL has been considered as an important pathway for complement activation, and multiple genetic backgrounds that limit MBL production may enhance susceptibility for unusual, recurrent, and serious gonococcal, meningococcal, staphylococcal, and pneumococcal infections, as documented, among others, in children and in patients with neutropenia or haematological cancer. ${ }^{1-8}$ However, a recent study indicates that only homozygosity for MBL deficiency increased the risk for pneumococcal disease in adults. ${ }^{7}$ Hence, the exact risk associated with genotypic or phenotypic MBL production deficiencies in general populations remains to be established. ${ }^{4}$ Nevertheless, our study does not exclude genetic MBL polymorphism predisposing to infection, even though the highly variable phenotypic MBL concentrations did not differ among the groups. ${ }^{12}$ Alternatively, the incidence of real MBL deficiency in our study population may be lower than the $8-15 \%$ in the white population reported by others and confirmed by our control data, even though $18 \%$ of our patients had MBL values below the arbitrary $0.1 \mu \mathrm{g} / \mathrm{ml}$, which may be in the range of concentrations found in heterozygous patients with MBL deficiency..$^{1-3}$ 5-7 $^{\text {However, }}$ we cannot exclude the possibility that we underestimated microbial infection in group 1 , in spite of a clinical focus and similar frequency of previous treatment with antibiotics as seen in the groups with documented microbial infection. The tendency for a rise in circulating MBL with increasing bacterial invasiveness and complement activation may, otherwise, point to an increasing acute phase response, because MBL may behave as an acute phase protein. ${ }^{15}$

\footnotetext{
"Mannan binding lectin deficiency does not seem to have prevented activation of the complement system"
} 


\section{Take home messages}

- Deficiency in the mannan binding lectin (MBL) pathway of complement activation did not predispose towards serious and invasive microbial infection in a heterogeneous population of hospitalised medical adults with new onset of fever

- This might not be the case in selected patients

- The results argue for a restricted policy for phenotypic and genetic MBL testing in patients without a history of serious or recurrent infections, or a positive family history

Although bacteraemia is a risk factor for shock and mortality during infection, ${ }^{9}$ there was no death following bacteraemia in the currently studied cohort, in spite of potentially harmful complement activation. Complement activation as a part of the innate host defence response may have either protected against death during bacteraemia, in spite of the alleged deleterious effects, or underlying diseases and sources of infection may have been less devastating than in the groups without infection or with local infection only. However, we could find no imbalances in favour of group 3 patients, except for different clinical sources and a higher frequency of Gram positive infections in the bacteraemic group, which may be associated with a relatively good prognosis. In any case, MBL deficiency does not seem to have prevented activation of the complement system. Our results are in line with the recent observation in Denmark $^{11}$ that variant $\mathrm{MBL}$ alleles did not predispose to serious pneumococcal infections in a general adult population. Moreover, in vitro complement activation by Neisseria gonorrhoeae does not necessarily require MBL. ${ }^{12}$

In conclusion, our results do not support the notion that MBL deficiency plays a role in susceptibility to microbial infection by impairing complement activation, in a heterogeneous population of hospitalised medical adults with new onset of fever. Obviously, this does not exclude a contribution in selected patients, as reported in the literature. ${ }^{356}$ Our findings may justify a restricted policy for phenotypic and genetic MBL testing, in patients without a history of serious or recurrent infections or a positive family history.

\section{Authors' affiliations}

A N Tacx, A B J Groeneveld, Department of Intensive Care, Vrije Universiteit Medical Centre, De Boelelaan 1117, 1081 HV Amsterdam, The Netherlands

M H Hart, L A Aarden, C E Hack, Sanquin Research at the Central Laboratory for Blood Transfusion Services, Amsterdam, The Netherlands

Correspondence to: Dr A B J Groeneveld, Department of Intensive Care, Vrije Universiteit Medical Centre, De Boelelaan 1117, 1081 HV Amsterdam, The Netherlands; johan.groeneveld@vumc.nl

Accepted for publication 4 August 2003

\section{REFERENCES}

1 Turner MW, Hamvas RMJ. Mannose-binding lectin: structure, function, genetics and disease associations. Rev Immunogenet 2000;2:305-22.

2 Petersen SV, Thiel S, Jensenius JC. The mannan-binding lectin pathway of complement activation: biology and disease association. Mol Immunol 2001;38:133-49.

3 Summerfield JA, Ryder S, Sumiya M, et al. Mannose binding protein gene mutations associated with unusual and severe infections in adults. Lancet 1995; $345: 886-9$

4 Summerfield JA, Sumiya M, Levin $M$, et al. Association of mutations in mannose binding protein gene with childhood infection in consecutive hospital series. Br Med J 1997;314:1229-32.

5 Neth O, Hann I, Turner MW, et al. Deficiency of mannose-binding lectin and burden of infection in children with malignancy: a prospective study. Lancet 2001;358:614-18

6 Peterslund NA, Koch C, Jensenius JC, et al. Association between deficiency of mannose-binding lectin and severe infections after chemotherapy. Lancet 2001;358:637-8.

7 Roy S, Segal S, Griffiths D, et al. MBL genotype and risk of invasive pneumococcal disease: a case-control study. Lancet 2002;359:1569-73.

8 Mullighan CG, Heatley S, Doherty K, et al. Mannose-binding lectin gene polymorphisms are associated with major infection following allogeneic hemopoietic stem cell transplantation. Blood 2002;99:3524-9.

9 Groeneveld ABJ, Bossink AWJ, Van Mierlo GJ, et al. Circulating inflammatory mediators in patients with fever: predicting bloodstream infection. Clin Diagn Lab Immunol $2001 ; 8: 1189-95$.

10 Crosdale DJ, Ollier WER, Thomson W, et al. Mannose binding lectin (MBL) genotype distributions with relation to serum levels in UK Caucasoids. Eur J Immunogen 2000;27:111-17.

11 Kronborg G, Weis N, Madsen HO, et al. Variant mannose-binding lectin alleles are not associated with susceptibility to or outcome of invasive pneumococcal infection in randomly included patients. J Infect Dis 2002;185:1517-20.

12 Gulati S, Sastry K, Jensenius JC, et al. Regulation of the mannan-binding lectin pathway of complement on Neisseria gonorrhoeae by $C_{1}$-inhibitor and $\alpha_{2}-$ macroglobulin. J Immunol 2002;168:4078-86. 